\title{
A HUMANOID-ROBOTICS METHOD FOR PREDICTING CONSTRUCTION WORK PERFORMANCE
}

\author{
Jonathan O'Brien
}

\author{
School of Civil and Environmental Engineering \\ University of New South Wales, Sydney, 2052, Australia \\ e-mail: j.obrien@unsw.edu.au
}

\begin{abstract}
:
A major obstacle to the refinement of modern construction engineering practice is the inability of the industry to exactly predict (in some theoretical rigorous manner) the beforethe-event performance of construction workers in the execution of particular tasks or to account for the actual method or systems of work employed or to allow for small changes in the task or in its solution technology. This paper addresses the (novel) worker-task performance prediction problem and seeks to solve it through the application of bumanoid robot programming and simulation techniques. The specific aim of this paper is to report on progress that has been made in the quantitative modelling of complex construction worker behaviour though humanoid robotics and virtual reality prototyping means and to present a proof of concept model of a working system.
\end{abstract}

Keywords:

Robotics, construction, simulation, virtual reality, digital people, ergonomics

\section{INTRODUCTION}

Modern construction and building actjvity is almost universally accomplished by direct or indirect bunan action in conjunction with the employment of various pieces of physical capital, implements, machines or other forms of apparatus. Relative to productivity and efficiency matters, studies of the use of physical capital and equipment, experimental studies of industrial and construction work perfornance clearly indicate that the efficiency of task performance is highly dependent on the method chosen by the worker to execute the task, the type of capital equipment that is deployed and the manner in which it is deployed [1-2]. Indeed, quite subtle changes in work methods and strategies and in team action strategies can result in major changes to output performance.]

\section{CURRENT METHODS FOR THE DESIGN OF CONSTRUCTION OPERATIONS}

To properly design and optimise industrial work performance systerns, one needs to be able to analyse and compare many alternative work strategies involving either purely manual or mixed mantmachine based work systems. Such work-systems design may be done in ones mind's eye (through the use of what Albert Einstein has referred to as "thought-experiments") or through the use of formal operations research methods and mathematical design procedures. To do fine design and to do detailed comparisons, the methods analysis needs to be done to a high level of resolution.[3]. Further, to properly predict construction work performance both the mental and physiological aspects of human task performance must be modelled. $[1,4]$

\subsection{The real-world experimental approach}

Perhaps the most fundamental approach to the engineering design of construction work-systems is to carry out a large series of real-world experiments to map "the deep structure of the problem space" $[5,6]$ then, to find a best method and thence to prototype the required task in-the-real. In most normal construction situations, however, this method is totally impractical since tasks are usually of a novel or one-off character and extensive pre-execution rehearsal is not-commercially feasible. Also, in the construction industry even what one might at first view as "simple" tasks are prohibitively expensive to rehearse. Most construction tasks are not at all simple and involve complex work performance with scores of variables, teans of workers and multiple mobile machines that interact with construction materials in a very complex mauner [7]. 


\subsection{The mental-world experimental approach}

Another fundamental approach to the engineering design of construction operations (albeit a somewhat derivative one [13]) is not based on physical experimentation but instead is based on search within an artificial (non-corporeal) world or "model" universe. In this virtual universe experimental approach, the real world is replaced by (simplified) models of the world that typically obey the same physics as the world. By experimenting on models of systems, predictions about the real world systems can be made. This method of design is very old in the history of engineering and is highly developed. Today, mathematical model-based process simulation comprises a powerful tool in modern engineering for the design and analysis of complex system performance. The use of simulation to virtually prototype complex real-work environments and task systems is now a highly developed engineering design art [8].

Where available, mathematical model based methods are almost universally preferred to realworld experimental design methods because of the very much lower costs.

\section{PRE-REQUISITES TO THE USE OF MATHEMATICAL-MODEL BASED SYSTEMS OF ENGINEERING DESIGN}

To effectively perform system simulation and virtual prototyping of dynamic interactive system however one needs to have what one might call "fullphysics" models of the task process and have a fullphysics model of the task environment. Mere empirical models and set logic (discrete event simulation methods) typically are inadequate for the, full-field, design task.

\section{MATHEMATICAL MODELS FOR THE PREDICTION OF HUMAN ACTION SEQUENCES}

To be able to apply the mathematical model based approach of traditional engineering design to humanbased construction and building operations, one must have an operational, full-physics, model of a person and be able to predict the physiological and time costs of any proposed specific sets of actions or action trajectories.

The model that has to be developed here is not the traditional static model of systems engineering but rather a model of staged decisional processes and of multi-stage systems which involves the use of an operator and an operand and involves matters of temporal and spatial logic.

To properly predict the physiological aspects of human action and human task performance one needs to have a full-physics dymanuics model of a person. In the model, one must be able to allow for the actual geometry and kinematics of human muscular-skeletal action as well as for the biomechanics and physiology of a specific person. Further, one must be able to allow for the processes of structural action and system dynamics. Moreover, to be accurate, the model must include such effects as muscle fatigue, limb-mass inertial effects, ranges of joint motion, strength and power-plant limits and so on. Further, for precise control of limb motions one must be able to calculate the torques and forces on all joints and to include body part acceleration and momentum factors.

Again, to the model of a person we must further add a model of the environment and thence set up some physics to permit these two discrete models to interact at the physical level $[8,10]$

\section{A NEW APPROACH TO PREDICTING CONSTRUCTION TASK PERFORMANCE THROUGH THE USE OF ROBOT MODELLING TECHNIQUES}

Because of the difficulty of applying standard mathematical model making techniques to predicting the consequences of complex skeins of human action and of the effects of decisional processes comprised of myriads of stages, a computationally-intensive mathematical modelling method based upon programmable robot notions and real-time modelling is hereby proposed to simulate the action of human workers.

Through the use of robotic modelling methods an operational model of a person can be developed and thence the resultant robot actor may programmed to execute "tasks" and do jobs within a physics based environment. By experimenting with "virtual people", various alternative designs for construction operations may be developed and considered.

The basic validity of this approach may be confirmed through the fact that real robots have been programmed to execute quite difficult human tasks. For example, real-robots now exist that can play ping-pong, hammer in a nail or shear a sheep.

\section{DEVELOPMENT OF A PRACTICAL CONSTRUCTION WORKER/TASK SIMULATION SYSTEM}

\subsection{Introduction}


The development and operation of humanoid-typ robots and simulating their operations in a interactive world of physical objects it a very difficu scientific and engineering exercise. However, $i$ principle, the approach is not that much differer from one that that has already been used by th author for the development of construction machiner simulators for environmental interactivity [10,8]. ] would seem that (provided than a realistic abstrac model of the system can be developed and that som computer graphics facility interface can be develope in some real-time language) then a general graphi output (virtual-reality type) simulator for buma: factors modelling can be developed.

\subsection{Kinematic models of the human body}

Despite its marvelous abilities and precisior mechanical engineers typically view the hurnan bod as a complex-jointed mechanism.. From a robc engineers perspective, a quite comprehensive study $c$ the mechanics of the human body has been carrie out by Rosheim [11]. His kinematic model of th buman body is reproduced as Figure 1. Also, a wealt] of extra detail about the specific mechanics of th human body is available from work in the fields o bio-mechanical engineering, artificial joir replacement, kinesiology and ergonomics.

\subsection{Interference checking requirements}

Apart from the need to accurately represent th physiology and kinematics of a person, in th development of task models there is also a need to $b$ able to represent a person's physical environment an to develop some computational way of modellin: intra-object interactions and collisions. Typically thi will require some solids-model of the world and som method of real-time (between part) interferenc checking [11].

\subsection{Methods for multi DOF robot programming}

Once one has a functionally progxammabl kinematically accurate, $\mathrm{N}$ degree-of-freedon humanoid mannequin that can interact with it environment in terms of the correct physics of th world, there still remains the problem of how $t$ program this robot so as to be able to do thing and $t$ operate in a proposed task-space. Two methods fo doing this work are potentially available. They are (j Direct pose-to-pose control of robots and (ii) Teac points and inverse kinematics methods

In the first method, any multi-DOF robot syster can be programmed by feeding into the model a fu series of joint coordinates. This direct, state-space control method however is potentially very tim consuming. A somewhat quicker approach is $t$ somewhat automate this process by giving the robot series of poses or positions and by providing machine-control software that will automatically generate intermediate values and thence morph the machine between poses.

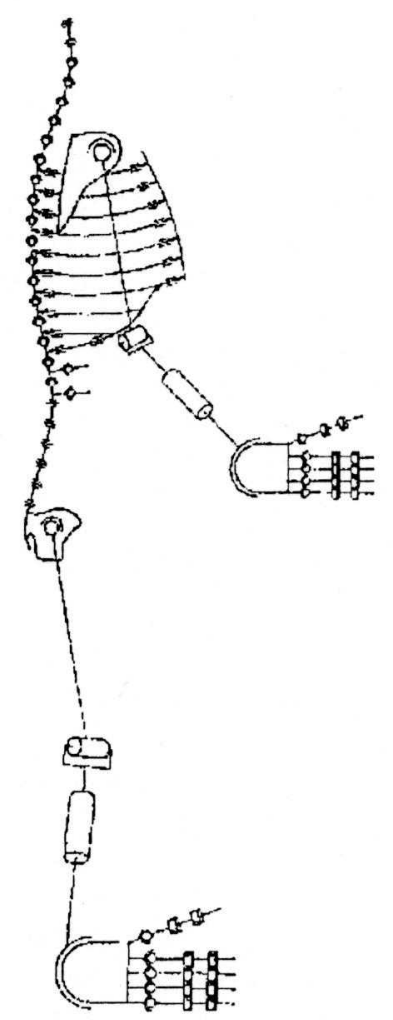

Figure 1 - A kinematic model of a person

In the second method, an alternative to robot programming in joint space is to use a highly simplified-method based around the idea of very structurally-stiff robot structures and teach-points. Where the inverse kinematics of the robot are known, or can be computed, one can present the robot with a desired end-points (also called tool point) and it will work out what joint angulations correspond to the end point. By the use of a series of teach points a robot can be programmed to operate along a predefined trajectory. This method is used extensively in the offline programming of industrial robots and welding machines [12].

Applying these ideas to the worker/task modelling problem, if we give a humanoid robot or programmable mannequin a series of teach point that correspond to a series of feet positions the robot can be programmed to morph between these points and hence can be made to "walk" along a predetermined line or path at a defined speed or velocity. Altematively, if the teach points are not planar the robot can be programmed to climb ladders or walk over rough terrain.

Similar ideas apply to the programming of the other limbs, neck, head and spine. 


\section{DEVELOPMENT OF A COMPUTATIONALLY-PRACTICAL HUMAN-WORK-OPERATIONS SIMULATOR}

\subsection{General}

The full-physics simulation of humans in interaction with the real-world is an extremely complex task that is probably well beyond the current state of the art. However in our attempts to develop these notions we can use some somewhat simplified models that sidestep many of the difficulties. Thus, for example is we assume that the hurnan body can be approximated by a stiff-structure and if we ignore some of the lesser degrees of freedom of the bodies joints e.g. the human bodies ability to twist at each spinal joint, to drop the hips and shoulders or to raise and lower individual scapula, some major simplifications in kinematics in a model-robot can be developed.

A further principal problem in the development of elaborate humanoid robots is in the development of the mathernatics to compute the state of the multiply redundant system and to do real-time structural analysis. This problem though can be reduced by adopting simplified machine kinematics.

\subsection{A simplified, stacked-robot, model of a person}

One approach to attempting to model human action through the use of an inverse kinematic teach point method, and the one that will be demonstrated here, is to develop what one might call a simplified stacked-industrial-robot model. If one models the human spine as serially linked robot operating from a fixed base (eg. the pelvis) then we can add the lower limbs and upper limbs as attachments stemming out of this base mechanism. Hands and robotic fingers can then be added to the limbs. Using this approach, one can develop a model of a robot that one might refer to as a 5 tier independently programmable system i.e. legs + spine + arms + hands + fingers + head. By programming these as separate machines, in task space or in joint coordinates, and through the use of teach points that can be computer generated, onthe-fly, the operation of a person in a work-space and performing an environmentally interactive task can be economically, and quite realistically, simulated.

\section{A PROTOTYPE VIRTUAL CONSTRUCTION WORKER MODELLING METHOD}

To demonstrate the feasibility of the use of the stacked-robot modelling method to model construction activity, a virtual person modelling system has been developed by the author that uses a commercial off-line robot programming system as it's kernel. The system has been adapted to accept computer control on-the-fly such that the virtual person's actions can be controlled interactively and in real-time. A quantitative output from the model, as to joint forces, energy consumption time taken to perform actions and so is available.

To illustrate the kind of output one might get, figure 2 shows a frame from an animation where a worker comes to a site, picks up a ladder and a rubber bladed wiper tool from a table and places the table against the glass window of a building. The worker then climbs up the ladder and proceeds to wash the window with the wiper tool using complex hand motions as in figure $2 \mathrm{~b}$. For the proof of concept demonstration, the robot was programmed by the use of a manually developed set of teach-points that were placed on the surface of the glass and on the ladder rungs to define the required tool path and feet paths. The robot software controller used inverse kinematics algorithms to compute all the body motions required and to program the actions of the virtual person in joint-coordinates.

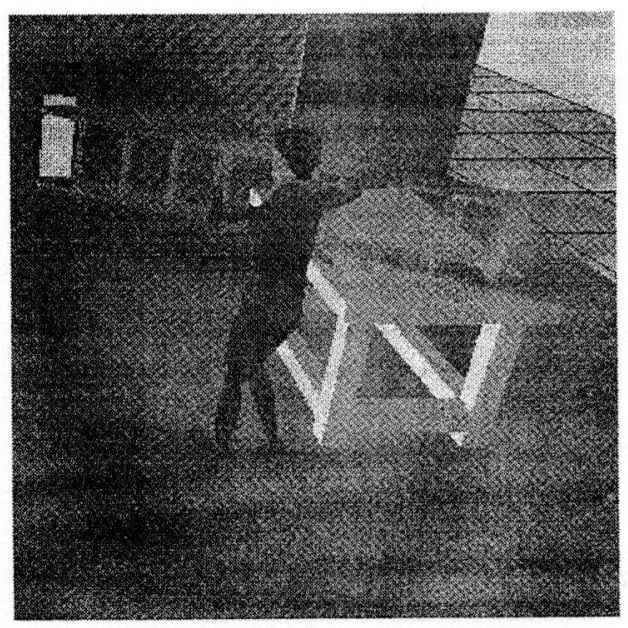

Figure 2a - Views of a "programmable person" executing a complex physical task under continuous computer control 


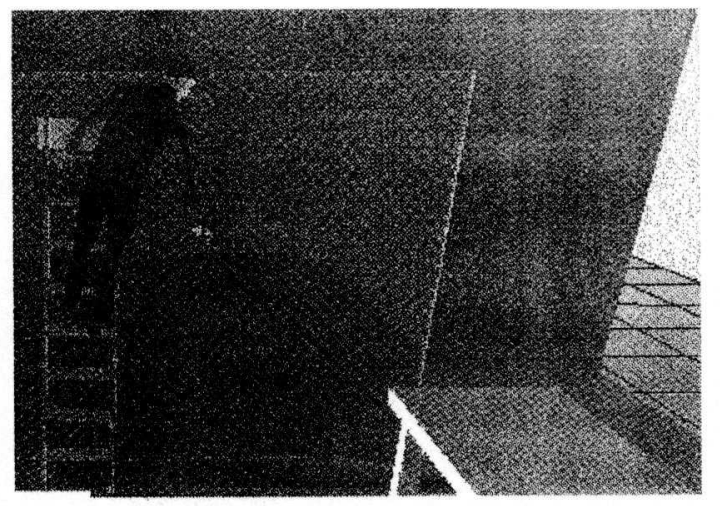

Figure $2 \mathrm{~b}$ - window wiping action

8.1 Modelling team operations and mixed man working-machine operations

A feature of the programmable-mannequin of virtual person method that has been developed here is that multi-robot system and mixed man-machine systems can be modelled. The machines can be fixed or mobile.

\section{POTENTIAL APPLICATIONS OF THIS TECHNOLOGY}

It has been proven through the use of robotic notions above and through the use of either manually generated or computer generated (artificial intelligence assisted ) teach points and or direct joint space programming that it is possible to prototype various task operations quite realistically. This system can then be used for example (a )To do very detailed methods engineering studies of task operations (b) For crew-work teaching and for work communication purposes (c) For the planning of work in confined spaces. (d) For detailed preplanning of work in inaccessible places and in time and safety critical operations. (e) For safety and $\mathrm{OH} \& \mathrm{~S}$ studies and for studies in work physiology and task ergonomics (f)For work-standards setting (g) For the cost and time estimating of complex one-off work systems

\section{CONCLUSIONS}

In this paper, a programmable mannequin or virtual-human method for the modelling of construction worker behaviour has been presented at a proof of concept level. It is believed that this approach is novel to the construction industry and represents a totally new-form of modelling technology for the prediction of industrial work performance within the building, construction and mining industry sectors. A number of potentially very valuable uses of this technology in scientific research and industrial $\mathrm{OH} \& \mathrm{~S}$ practice have been identified. The applications and developments will extend with improvements in mechanical models and in taskspace programming method. Whilst the approach demonstrated is only in its infancy, a bright future for this kind of technology may be predicted

\section{ACKNOWLEDGEMENTS}

The author is much indebted to Mike Goodwin of Deneb, Australia for the programming work behind figure 2.

\section{REFERENCES}

[1] O'Brien J.B. and Compton R "Field Studies of the Cognitive Processes involved in Construction Activity" Proc. 4th Intl. Symposium on Robotics and Artificial Intelligence in Building Construction, Building Research Station, Technion, Haifa Israel, June 22-25 1987, pp322342 .

[2] e.g. Barnes R. M "Motion and Time Study", Wiley 1980

[3] CIRIA "Development and trials of alternative methods of bricklaying" Constrcution Industry Research and Information Association, U.K., Report 42

[4] Fleishman Edwin A. (ed). Human performance and productivity" Vols I-III, Lawrence Erlbaum publishers. 1982.

[5] Newell A. and Simon H. A. "Human Problem Solving' Prentice Hall 1972

[6] Ashby W. R. "Design for a brain" 2nd ed. Chapman and Hall, London, ISBN 0-412-20090-2

[7] O'Brien, J. B. “ A Unified Theory of Construction Robotics" Proc 15th ISARC Munich, Germany 1998, pp. 191-200.

[8] Wakefield, R. R. and O'Brien, J. B. (1994). "A virtual reality" type system for construction automation system development" ISARC XI, 1994, pp 239-247

[9] cf. for example the Caterpillar Tractor Company's haul unit travel time prediction methods.

[10] Wakefield R. and O'Brien J.B. "Real Time, Visual Output Simulation - A New Process Tool For Construction Planners And Managers" Proc National Construction and Management Conference, Sydney, Feb 17-18 1994, Wakefield $\mathrm{R}$ and Carmichael D. (eds) IEAust/UNSW pp227242. ISBN 08584143333

[11] Mark E. Rosheim "Robot Evolution - the development of Anthrobotics". John Wiley \& Sons 1994, 419 pages

[12] McKerrow Philip J "Introduction to Robotics" Addison Wesley 1991

[13] Mach E. " The Science of Mechanics - a critical and historical account of its development. 6th ed. Open Court Pub. Co. 1960. 